\title{
RESEARCH
}

Open Access

\section{Impact of clinical and sociodemographic factors on fatigue among patients with substance use disorder: a cohort study from Norway for the period 2016-2020}

Jørn Henrik Vold ${ }^{1,2^{*}}$ (D), Rolf Gjestad ${ }^{3}$, Christer F. Aas ${ }^{1,2}$, Fatemeh Chalabianloo ${ }^{1,2}$, Svetlana Skurtveit ${ }^{4,5}$, Else-Marie Løberg ${ }^{1,3,6}$, Kjell Arne Johansson ${ }^{1,2}$, Lars Thore Fadnes ${ }^{1,2}$ and for the INTRO-HCV Study Group

\begin{abstract}
Background: The impact of clinical and sociodemographic factors on fatigue remains unknown among patients with substance use disorders (SUD). This study aims to evaluate fatigue among patients with SUD using a nine-item fatigue severity scale (FSS-9) and identify the impact that clinical and sociodemographic factors - such as injecting substance use, chronic infectious diseases, liver fibrosis, opioid agonist therapy (OAT), debt difficulties, and housing situation - have on fatigue.
\end{abstract}

Methods: We used data from a cohort of patients with SUD in Norway with annual health assessments surveying FSS-9 and some clinical and sociodemographic factors. A total of 915 FSS-9 measurements were collected from 654 patients during the period 2016-2020. We defined baseline as the first annual health assessment when the health assessments were listed chronologically. Time was defined as years from baseline. We used a linear mixed model to analyse whether the clinical and sociodemographic factors affected the FSS-9 sum score, presented with beta coefficients ( $\beta$ ) with 95\% confidence intervals (CI).

Results: The mean sum score of the FSS-9 was 43 (standard deviation: 16) at baseline. Females compared with males (adjusted mean difference of FSS-9 sum score: 4.1, 95\% CI: 1.3-7.0), having debt difficulties compared with having no debt difficulties (2.9;0.4-5.3), and frequent use of benzodiazepines (5.7;3.0-8.4) or amphetamines (-5.0;8.0- -2.0) compared to less frequent or no use of these substances changed the FSS-9 baseline sum score. The other clinical and sociodemographic factors did not predict any clinically relevant change in the FSS-9 sum score from baseline to the following health assessments.

Conclusion: Patients with SUD suffer from high levels of fatigue. Female patients, patients with debt difficulties, and those with extensive use of benzodiazepines are at particular risk of being fatigued. This should be taken into consideration when planning health services.

Keywords: Substance-related disorders, Fatigue, Fatigue severity scale, Quality of life, Comorbidities, Illicit drugs, Viral human hepatitis, HIV, Kidney disease

\footnotetext{
* Correspondence: jorn.vold@uib.no

'Department of Addiction Medicine, Haukeland University Hospital, Jonas Lies vei 65, N-5021 Bergen, Norway

${ }^{2}$ Department of Global Public Health and Primary Care, University of Bergen, Bergen, Norway

Full list of author information is available at the end of the article
}

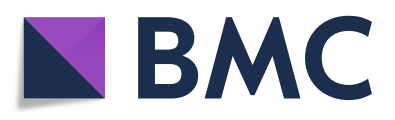

(- The Author(s). 2020 Open Access This article is licensed under a Creative Commons Attribution 4.0 International License, which permits use, sharing, adaptation, distribution and reproduction in any medium or format, as long as you give appropriate credit to the original author(s) and the source, provide a link to the Creative Commons licence, and indicate if changes were made. The images or other third party material in this article are included in the article's Creative Commons licence, unless indicated otherwise in a credit line to the material. If material is not included in the article's Creative Commons licence and your intended use is not permitted by statutory regulation or exceeds the permitted use, you will need to obtain permission directly from the copyright holder. To view a copy of this licence, visit http://creativecommons.org/licenses/by/4.0/. The Creative Commons Public Domain Dedication waiver (http://creativecommons.org/publicdomain/zero/1.0/) applies to the data made available in this article, unless otherwise stated in a credit line to the data. 


\section{Background}

Patients with Substance Use Disorders (SUD) suffer from a broad range of health-related difficulties that may contribute to fatigue [1-3]. Fatigue presents itself as a persistent and overwhelming feeling of exhaustion and loss of energy. The condition is mainly associated with chronic diseases and may mitigate treatment adherence and exacerbate comorbid disorders $[4,5]$. In SUD populations, a myriad of external factors can interact with fatigue and affect these patients' general well-being [6-8]. Injecting substance use, internal organ dysfunctions (predominantly kidney and liver diseases), mental disorders, as well as low income, unemployment, and homelessness are some of the external factors that interact with fatigue. Despite this, relatively little attention has been paid to the extent of fatigue and how much various external factors influence fatigue among patients with SUD. Therefore, understanding the key factors affecting fatigue is essential to improve treatment outcomes and adherence in this population.

Fatigue is associated with several sociodemographic and clinical factors. Among patients with the Hepatitis $\mathrm{C}$ Virus (HCV) infection, 50-70\% have reported fatigue [9-11], while $33-88 \%$ of those with the Human Immunodeficiency Virus (HIV) infection have presented the same symptom [12]. A more uncertain prevalence of fatigue is seen among patients with the Hepatitis B Virus (HBV) $[13,14]$. In addition, females, patients with lower educational levels, and those with opioid use disorders undergoing Opioid Agonist Therapy (OAT) with methadone or buprenorphine generally have a greater risk of fatigue $[15,16]$. Disentangling the effects of the potential factors influencing fatigue in patients with SUD is essential for individualised treatment and developing clinical guidelines.

Fatigue is a subjective concept, and various definitions and instruments are used in the literature to capture it, which makes interpretations more complicated [17-19]. The nine-item fatigue severity scale (FSS-9) is a wellknown questionnaire used to quantify fatigue treatment effects. It shows excellent validity and reliability across various chronic neurological and infectious diseases, such as multiple sclerosis [17], HCV infection [20], stroke [5], and Parkinson's disease [21]. The fact that FSS-9 shows a high consistency across various chronic diseases makes it particularly suitable to estimate fatigue among patients suffering from SUD with complex and challenging comorbidities.

Thus, this prospective cohort study aims to investigate fatigue using the nine-item Fatigue severity scale (FSS-9) among patients with substance use disorders (SUDs) and predict the impact of sociodemographic and clinical factors on FSS-9, including educational level, housing situation, debt difficulties, chronic infectious diseases, injecting substance use, substance use, liver fibrosis, and kidney disease. Moreover, we estimate:

1) using annual health assessments, the FSS-9 sum score and whether and to what extent the sociodemographic and clinical factors impact this score;

2) the impact of sociodemographic and clinical factors on changes in the FSS-9 sum score from the first health assessment to the following annual health assessments;

3) for two separate subgroups - patients receiving methadone as opioid agonist therapy (OAT) and those receiving buprenorphine as OAT - the FSS-9 sum score, whether and to what extent the sociodemographic and clinical factors affect the FSS-9 sum score at baseline, and any changes in the FSS-9 sum score from the first health assessment to the following annual health assessments.

\section{Methods}

\section{Data source}

We used data from a cohort nested to the INTRO-HCV trial on patients with SUD in Bergen and Stavanger, Norway [22]. We collected data from May 2016 to January 2020, and recruited patients on OAT from outpatient clinics in Bergen and Stavanger, as well as patients with various SUDs receiving primary healthcare from the municipality clinics in the city of Bergen.

\section{Data collections}

All included patients were assessed yearly with a health assessment, including FSS-9 measurements, sociodemographic data, and current substance use. Additionally, blood samples and liver fibrosis measurements using transient elastography were conducted. We collected all data in a health register using electronic data collection software (Checkware ${ }^{\oplus}$ ) under research nurses' supervision. All the clinical data, including information regarding OAT, OAT medication, substance use, and possible comorbid clinical conditions, were collected from the electronic medical record.

\section{Study sample}

We included 915 FSS-9 measurements from 654 patients in the study period. In total, 225 had follow-up data and conducted the health assessment, including the FSS-9 questionnaire, twice $(n=188)$ or thrice $(n=37)$, providing 487 repeated measurements. The median time interval between the baseline health assessments, and any subsequent assessments in the same patients, including FSS-9 measurements, was 11 months (interquartile range (IQR): 9-14) (Additional file 1). 


\section{Measuring fatigue}

We measured fatigue during the last week using FSS-9, including items considering: mental and physical functioning, motivation, carrying out duties, and interference with work, family, or social life. An FSS-9 measurement was completed when all nine items in the questionnaires were entirely conducted during an annual health assessment. The FSS-9 items were answered on a Likert scale - ranging from 1 (no fatigue) to 7 (worst fatigue) - that demonstrates the fatigue level. A high score of FSS-9 items notes a high level of fatigue, while a mean FSS-9 item score greater than 4.0 revealed severe fatigue. The data collection software only allowed valid responses to each question and prompted empty questions before submission to minimise missing data. The FSS-9 was also translated and back-translated from the US-English version into Norwegian by qualified native Norwegianspeaking translators (Additional file 2) [23].

\section{Measuring liver stiffness and assessing blood samples}

We assessed liver stiffness using transient elastography $\left(\right.$ Fibroscan $\left.^{\circ}\right)$ to reveal liver fibrosis and cirrhosis. The elastography was reported as a median score of 10 measurements conducted by research nurses. A liver stiffness above 10 kilopascals $(\mathrm{kPa})$ was defined as liver fibrosis, while a value above $12.5 \mathrm{kPa}$ indicated liver cirrhosis [24]. We also collected blood samples, including hemoglobin, thrombocytes, $\mathrm{C}$ - reactive protein, aspartate aminotransferase, estimated glomerular filtration rate, hepatitis B surface antigen, HIV antigen/antibodies, $\mathrm{HCV}$ antibodies, and HCV polymerase chain reaction (HCV PCR) during the annual health assessment. Liver stiffness was estimated by calculating the AST to platelet ratio index (APRI) score and using transient elastography (Fibroscan ${ }^{\circ}$ ) (Additional file 3). Moreover, the hematological and biochemical samples were analysed to detect anemia (Hemoglobin), infection or inflammation (C - reactive protein), kidney disease (estimated glomerular filtration rate), liver disease (APRI), or chronic infectious diseases (HIV, HCV, and HBV), which could affect the FSS-9 score. Both elastography and blood samples were examined annually and simultaneously when conducting the annual health assessments. We analysed the blood samples at the Department of Laboratory Medicine, Haukeland University Hospital, Bergen, Norway, and at the Department of Medical Biochemistry and Microbiology, Stavanger University Hospital, Stavanger, Norway (accredited by ISO-standard 15,189).

Definition of study variables, including sociodemographic and clinical factors

We defined baseline for patients as the first annual health assessment that included an FSS-9 measurement when we listed the health assessments chronologically.
We dealt with each FSS-9 measurement as a sum score by summarising the value (one to seven) from each item and as a mean score calculated by dividing the sum score by nine (nine items). We defined being on OAT according to whether patients received buprenorphine or methadone (OAT opioids) at baseline. Further, in accordance with the World Health Organization's standards, we calculated the daily dose of received OAT opioids as a ratio between the received dose per day divided by the expected mean dose of OAT opioids (buprenorphine $18 \mathrm{mg}$, buprenorphine-naloxone 18/4.5 $\mathrm{mg}$ or methadone $90 \mathrm{mg}$ ) [25]. We categorised educational level into five groups: 'not completed primary school,' 'completed primary school (nine years),' 'completed high school (12 years),' 'three or fewer years of college or university' or 'more than three years of college or university.' Patients' housing situations in the 30 days prior to the FSS-9 measurement were classified into two groups: "stable" and "unstable." The latter category involved patients who had lived on the street, in a homeless shelter, or with family and friends during the past 30 days. Others who had a more permanent residence were classified as having a stable housing situation. Debt difficulties were defined as striving with paying off legal or illegal debt due to a constrained private economy. We set 'injecting substance use' as having injected at any time during the past 12 months, whereas frequent substance use was categorised as consuming at least one of the substance groups, including 'benzodiazepines or $\mathrm{z}$ hypnotics,' 'cannabis,' 'stimulants (amphetamines or cocaine),' 'alcohol,' and 'heroin or other illicit opioids', more than weekly during the 12 months prior to a health assessment. Patients who did not use substances or used them less than weekly during the past 12 months were categorised as having 'no frequent use of substance'. Having chronic infectious diseases was defined as detecting HCV PCR (HCV), hepatitis B surface antigen (HBV), or HIV antigen/antibodies (HIV) in the blood samples. For HCV PCR, we used the Helmert contrast in order to classify patients into two groups - transmitted and nontransmitted - and further into two subgroups: whether patients have a low viral load $(<800,000 \mathrm{IU} / \mathrm{ml})$ or high viral load $(\geq 800,000 \mathrm{IU} / \mathrm{ml})$. By this two-fold division, we investigated whether the level of viral HCV load was associated with changes in the fatigue level.

\section{Statistical analyses}

We used Stata/SE 16.0 (StataCorp, TX, USA) for descriptive analysis and IBM SPSS version 26.0 for expectation-maximisation imputation and linear mixed model analyses. The threshold for statistical significance was set to $P<0.05$ for all analyses unless otherwise stated. In all analyses, we defined time as years from baseline. 
We dealt with any missing values concerning sociodemographic and clinical factors - such as educational level, housing situation, debt difficulties, receiving OAT, OAT opioid dose ratio, injecting substance use, substance use, and the results of defined blood samples and transient elastography - as 'missing at random' when running expectation-maximisation imputation. We identified missing values in $2.6 \%$ in these factors and all were replaced with estimated values by imputation.

The FSS-9 sum score at baseline was calculated by summarising the nine items' points. Linear mixed model analyses were used to investigate whether the sociodemographic and clinical factors affected the FSS-9 sum score and to what extent they impacted any changes in the score from baseline to following the health assessments. First, the factor variables were analysed separately as outcome variables as a function of the time (time from baseline). We did not identify substantial significant changes in the sociodemographic and clinical factors between the annual health assessments (data not shown). Thus, baseline levels were used as stable predictors in the prediction of the level and changes in FSS-9. We specified the linear mixed models as a random intercept fixed slope regression model. The estimator was set to Restricted Maximum Likelihood. To explore whether predictors predicted changes in outcome, the interactions between these factors and time were added to the model. The full information maximum likelihood ensured that all available FSS-9 sum score measurements were used. Additionally, we presented sub-group analyses for OAT patients using methadone or buprenorphine, respectively. For these analyses, we added the OAT opioid ratio as a predictor. The potential correlations between sociodemographic and clinical factors and fatigue are presented in Additional file 4. We performed a sensitivity analysis by adding Bonferroni corrected $p$ values to adjust for Type I errors in all analyses.

\section{Ethics approval and consent to participate}

The study is reviewed and approved by the Regional Ethical Committee for Health Research West, Norway (REK Vest 2017/51). Each patient provided written informed consent prior to enrolling in the study.

\section{Results}

\section{Patients characteristics at baseline}

Seventy-one percent of patients were male, and the mean age was 43 years (standard deviation (SD): 11 years) at baseline (Table 1). Six percent had not completed primary school, or $44 \%$ had primary school as their highest educational level. $82 \%$ received OAT, of which $60 \%$ received buprenorphine or buprenorphine-naloxone as an OAT opioid. Further, 13\% had an unstable housing situation in the last 30 days leading up to the FSS-9 measurement. $73 \%$ had used at least one substance weekly during the past 12 months.

\section{FSS-9 sum scores at baseline}

The mean sum score for the FSS-9 was 43 (SD: 16), representing a mean score for the FSS-9 items of 4.8 (2.6) (Table 2). A total of $69 \%$ of patients had severe fatigue. The mean FSS-9 sum score was slightly leftskewed (skewness: -0.7 ) and tended towards a flattened distribution (kurtosis: 2.4).

The mean scores for the FSS-9 were 43 (SD: 16) for patients receiving methadone and 43 (17) for those using buprenorphine (Additional file 5), corresponding to a mean score for the FSS-9 items of 4.8 (1.8) for patients receiving methadone and 4.7 (1.9) for those using buprenorphine. Severe fatigue was identified in $77 \%$ of patients receiving methadone and $67 \%$ of those using buprenorphine. In these two sub-groups, the FSS-9 sum scores were slightly leftskewed (skewness: - 1.0 (methadone group), - 0.6 (buprenorphine group)) and flattened distributed (kurtosis: 3.0 (methadone group), 2.2 (buprenorphine group)).

\section{The sociodemographic and clinical factors' impact on the FSS-9 sum score at baseline and the factors' influence on changes in the FSS-9 sum score from baseline to the following annual health assessments}

At baseline, we found that the FSS-9 sum score was higher for females than males (adjusted mean FSS-9 sum score difference: 4.1, CI 1.3;7.0, $p=0.005$ ), for patients with debt difficulties compared with those without debt difficulties (2.9, CI $0.4 ; 5.3, p=0.022$ ), and for patients with frequent benzodiazepine use compared with those with less frequent or no use $(5.7$, CI 3.0;8.4, $p<0.001)$ (Table 3). In contrast, the FSS9 sum score was lower for patients with frequent stimulant use than those with less frequent or no use (-5.0, CI -8.0;$2.0, p=0.001)$. Moreover, we saw a small non-clinical significant reduction of the FSS-9 sum score from baseline to the following annual health assessments for patients with frequent benzodiazepine use compared to those with less frequent or no use $(-4.4, \mathrm{CI}-8.2 ;-0.7, p=0.021)$ and for patients having significant liver fibrosis or cirrhosis measured by transient elastography compared with those with nonsignificant fibrosis or normal liver stiffness (- 5.5, CI -9.9;-1.0, $p=0.016)$. With Bonferroni corrected $p$-values, we only found that patients with frequent benzodiazepine or stimulant use compared with those with less frequent or no use of these substances changed the fatigue levels at baseline.

\section{The sociodemographic and clinical factors' impact on changes in the FSS-9 sum score from baseline to the following annual health assessments among patients on OAT}

Among patients receiving methadone as an OAT opioid, we found that the FSS-9 sum score was higher for 
Table 1 Sociodemographic and clinical characteristics at baseline for all patients and for patients with more than one annual health assessment

\begin{tabular}{|c|c|c|}
\hline & $\begin{array}{l}\text { All } \\
\text { patients } \\
(N=654)\end{array}$ & $\begin{array}{l}\text { Patients with } \\
\geq 2 \text { health assessments } \\
(N=225)\end{array}$ \\
\hline \multicolumn{3}{|l|}{ Age (years), $n(\%)$} \\
\hline $18-29$ & $81(12)$ & $23(10)$ \\
\hline $30-39$ & $185(28)$ & $63(28)$ \\
\hline $40-49$ & $205(31)$ & $75(33)$ \\
\hline $50-59$ & $148(23)$ & $53(24)$ \\
\hline$\geq 60$ & $35(5)$ & $11(5)$ \\
\hline Mean (SD) & $43(11)$ & $44(10)$ \\
\hline \multicolumn{3}{|l|}{ Gender, n (\%) } \\
\hline Male & $461(71)$ & $170(76)$ \\
\hline Female & $193(29)$ & $55(24)$ \\
\hline \multicolumn{3}{|l|}{ Highest educational level, n (\%) } \\
\hline Not completed primary school & $40(6)$ & $15(7)$ \\
\hline Completed primary school (9years) & $286(44)$ & $105(47)$ \\
\hline Completed high school (12 years) & $259(40)$ & $81(36)$ \\
\hline$\leq 3$ years of college or university & $57(9)$ & $20(9)$ \\
\hline$>3$ years of college or university & $12(2)$ & $<5(2)$ \\
\hline Receiving opioid agonist therapy, $n$ (\%) & $537(82)$ & $205(91)$ \\
\hline \multicolumn{3}{|l|}{ OAT opioid (\%) } \\
\hline Methadone & 209 (39) & $96(43)$ \\
\hline Buprenorphine/Buprenorphine-naloxone & $321(60)$ & $107(48)$ \\
\hline OAT opioid dose ratio (median $(I Q R))^{\mathrm{a}}$ & $0.9(0.8-1.1)$ & $1.0(0.9-1.1)$ \\
\hline \multicolumn{3}{|l|}{ Housing situation the past 30 days, $n$ (\%) } \\
\hline Stable ${ }^{b}$ & $569(87)$ & $203(90)$ \\
\hline Unstable & $85(13)$ & $22(10)$ \\
\hline Injected substances the past 12 months, $n$ (\%) & $338(56)$ & $116(52)$ \\
\hline \multicolumn{3}{|l|}{ Frequent substance use the past 12 months, $n(\%)^{d}$} \\
\hline Alcohol & $154(26)$ & $56(25)$ \\
\hline Benzodiazepines & 238 (39) & $87(39)$ \\
\hline Cannabis & $313(52)$ & $124(55)$ \\
\hline Opioids & $97(16)$ & $27(12)$ \\
\hline Stimulants (amphetamines and cocaine) & $176(29)$ & $60(27)$ \\
\hline \multicolumn{3}{|l|}{ Chronic infectious diseases, $n$ (\%) } \\
\hline Hepatitis C virus infection & $315(48)$ & $184(82)$ \\
\hline Low virulent $(<800,000 \mathrm{IE} / \mathrm{ml})$ & $168(25)$ & $92(41)$ \\
\hline High virulent ( $\geq 800,000 \mathrm{IE} / \mathrm{ml})$ & $147(22)$ & $92(41)$ \\
\hline Hepatitis B virus infection & $5(0)$ & $<5(<1)$ \\
\hline Human immunodeficiency virus & $<5(<1)$ & $<5(<1)$ \\
\hline \multicolumn{3}{|l|}{ Hematological and biochemical samples, median (IQR) } \\
\hline Hemoglobin $(g / d l)$ & $14(13-15)$ & $14(13-15)$ \\
\hline Estimated glomerulus filtration rate $\left(\mathrm{ml} / \mathrm{min} / 1.73 \mathrm{~m}^{2}\right)$ & $104(89-122)$ & $105(91-124)$ \\
\hline C-reactive protein (mg/L) & $4(1-9)$ & $3(1-8)$ \\
\hline Aspartate transaminase $(\mathrm{U} / \mathrm{L})$ & $31(23-50)$ & $40(30-65)$ \\
\hline
\end{tabular}


Table 1 Sociodemographic and clinical characteristics at baseline for all patients and for patients with more than one annual health assessment (Continued)

\begin{tabular}{|c|c|c|}
\hline & $\begin{array}{l}\text { All } \\
\text { patients } \\
(N=654)\end{array}$ & $\begin{array}{l}\text { Patients with } \\
\geq 2 \text { health assessments } \\
(N=225)\end{array}$ \\
\hline \multicolumn{3}{|l|}{ Liver stiffness, median (IQR) } \\
\hline Transient elastography (kPa) & $5(4-7)$ & $6(5-8)$ \\
\hline Aspartate transaminase to platelets ratio index & $0.3(0.2-0.6)$ & $0.4(0.3-0.8)$ \\
\hline
\end{tabular}

females than males $(7.3, \mathrm{CI} 2.5 ; 12.2, p=0.003)$, for patients having debt difficulties compared with those not having debt difficulties $(4.9$, CI $0.7 ; 9.1, p=0.023)$, for patients having frequent benzodiazepine use compared with those having less frequent or no use (6.0, CI 1.6; 10.5, $p=0.008$ ), and for patients with a high $\mathrm{HCV}$ viral load compared with those with a low HCV viral load (31.5, CI 1.5;61.5, $p=0.040$ ) at baseline (Additional file 6). Among patients receiving buprenorphine as an OAT opioid, we found that patients with frequent alcohol use had higher the FSS-9 sum score (4.8, CI 0.2;9.3, $p=$ 0.039), while patients with frequent stimulant use had lower the FSS-9 score $(-5.0$, CI -9.9;-0.1, $p=0.047)$ compared with patients with less frequent or no use of these substances at baseline (Additional file 7). For both subgroups, no sociodemographic and clinical factors were clinically associated with substantial changes in the FSS-9 sum score from baseline to the following annual health assessments. With Bonferroni corrected $p$-values, we did not identify any predictors that changed the fatigue score at baseline and between the annual health assessments.

\section{Discussion}

This study showed that $69 \%$ of SUD patients had severe fatigue symptoms. The sociodemographic and clinical factors that substantially contributed to higher fatigue scores at baseline were females compared with males (four points), frequent benzodiazepine use compared with less frequent or no use (six points), and debt difficulties compared with no debt difficulties (three points). However, the fatigue score was five points lower for patients with frequent stimulant use than those with less frequent or no use. For patients using buprenorphine as an OAT opioid, we found five points lower fatigue score for patients with frequent stimulant use and five points higher fatigue score for patients with frequent alcohol

Table 2 Mean (Standard deviation (SD)) item scores for single items on FSS-9 at baseline and follow-up

\begin{tabular}{|c|c|c|}
\hline & Baseline $(N=654)$ & Follow-up $(N=225)$ \\
\hline \multicolumn{3}{|c|}{ FSS-9 } \\
\hline 11: My motivation is lower when I am fatigued & $5.4(2.0)$ & $5.6(2.0)$ \\
\hline 12: Exercise brings on my fatigue & $4.7(2.1)$ & $5.0(2.0)$ \\
\hline 13: I am easily fatigued & $4.5(2.1)$ & $4.8(2.1)$ \\
\hline 14: Fatigue interferes with my physical functioning & $4.9(2.1)$ & $5.1(2.0)$ \\
\hline 15: Fatigue causes frequent problems for me & $4.4(2.2)$ & $4.5(2.2)$ \\
\hline 16: My fatigue prevents sustained physical functioning & $4.6(2.2)$ & $4.4(2.2)$ \\
\hline 17: Fatigue interferes with carrying out certain duties and responsibilities & $5.0(2.1)$ & $5.0(2.1)$ \\
\hline I8: Fatigue is among my three most disabling symptoms & $4.6(2.3)$ & $4.8(2.3)$ \\
\hline 19: Fatigue interferes with my work, family, or social life & $4.9(2.2)$ & $4.6(2.3)$ \\
\hline Mean score of all items & $4.8(1.8)$ & $4.9(1.7)$ \\
\hline Sum score of all items & $43.2(15.9)$ & $43.8(15.2)$ \\
\hline
\end{tabular}

Follow-up: FSS-9 score on the last health assessment during the study period among patients with two or more annual health assessments; FSS-9 nine-item fatigue severity scale (Likert scale ranging from 1 (no fatigue) to 7 (worst fatigue)), I Item, SD standard deviation 
Table 3 Linear mixed model of fatigue (FSS-9) adjusted for sociodemographic and clinical factors ( $N=654)$

\begin{tabular}{|c|c|c|c|c|}
\hline \multicolumn{3}{|l|}{ Fixed effects } & \multirow{2}{*}{\multicolumn{2}{|c|}{ Time trend (per year) }} \\
\hline & \multicolumn{2}{|l|}{ Effect estimate } & & \\
\hline & Estimate $(95 \% \mathrm{Cl})$ & $p$-value & Slope $(95 \% \mathrm{Cl})$ & $p$-value \\
\hline FSS-9 sum score & $42(26-58)$ & $<.001$ & $3.6(-23.5-30.7)$ & 0.792 \\
\hline Female & $4.1(1.3-7.0)$ & 0.005 & $-0.3(-4.5-3.8)$ & 0.877 \\
\hline Age per 10 years ${ }^{1)}$ & $0.2(-1.0-1.4)$ & 0.755 & $-0.2(-2.1-1.7)$ & 0.844 \\
\hline Educational level & $-1.1(-2.6-0.3)$ & 0.132 & $-0.3(-2.3-1.7)$ & 0.754 \\
\hline Unstable housing situation & $0.0(-3.7-3.7)$ & 0.992 & $2.6(-3.6-8.8)$ & 0.408 \\
\hline Debt difficulties & $2.9(0.4-5.3)$ & 0.022 & $-0.2(-3.8-3.3)$ & 0.898 \\
\hline Injecting substance use & $-0.1(-2.9-2.7)$ & 0.944 & $-0.7(-4.6-3.3)$ & 0.740 \\
\hline \multicolumn{5}{|l|}{ Frequent use of substances } \\
\hline Benzodiazepines & $5.7(3.0-8.4)$ & $<.001^{a}$ & $-4.4(-8.2--0.7)$ & 0.021 \\
\hline Alcohol & $1.8(-1.1-4.6)$ & 0.221 & $0.6(-3.5-4.7)$ & 0.776 \\
\hline Cannabis & $1.2(-1.4-3.8)$ & 0.356 & $1.8(-1.7-5.3)$ & 0.309 \\
\hline Opioids & $3.3(-0.3-6.9)$ & 0.069 & $-4.6(-10.8-1.7)$ & 0.149 \\
\hline Stimulants ${ }^{2)}$ & $-5.0(-8.0--2.0)$ & $0.001^{a}$ & $2.1(-2.1-6.3)$ & 0.327 \\
\hline \multicolumn{5}{|l|}{ Chronic infectious diseases } \\
\hline Hepatitis B virus infection & $3.3(-10.4-16.9)$ & 0.638 & $-2.6(-16.8-11.5)$ & 0.715 \\
\hline \multicolumn{5}{|l|}{ Hepatitis C virus infection } \\
\hline - Detected & $3.0(-5.4-11.4)$ & 0.484 & $0.7(-18.7-20.1)$ & 0.941 \\
\hline - Low vs. high viral load & $-0.4(-10.3-10.9)$ & 0.948 & $-7.0(-17.1-3.0)$ & 0.169 \\
\hline HIV & $-0.1(-15.3-15.5)$ & 0.994 & $13.0(-6.8-32.7)$ & 0.197 \\
\hline \multicolumn{5}{|l|}{ Liver stiffness } \\
\hline Transient elastography per $10 \mathrm{kPa}$ & $1.2(-1.6-4.0)$ & 0.391 & $-5.5(-9.9--1.0)$ & 0.016 \\
\hline APRI score per 1 unit & $0.5(-0.6-1.5)$ & 0.378 & $1.4(-0.9-3.6)$ & 0.230 \\
\hline \multicolumn{5}{|c|}{ Hematologic and biochemical blood samples (continuous variables) } \\
\hline Hemoglobin per 1 unit (g/dL) & $-0.3(-1.1-0.6)$ & 0.513 & $0.3(-0.9-1.5)$ & 0.622 \\
\hline eGFR per 30 units ( $\left.\mathrm{ml} / \mathrm{min} / 1.73 \mathrm{~m}^{2}\right)$ & $0.0(-2.0-0.9)$ & 0.453 & $0.0(-2.0-1.9)$ & 0.973 \\
\hline CRP per 10 units (ml/L) & $-0.1(-0.6-0.7)$ & 0.848 & $0.0(-0.1-0.2)$ & 0.682 \\
\hline
\end{tabular}

The table displays a linear mixed model analysis (Restricted Maximum Likelihood regression) evaluating sociodemographic and clinical factors' (predictors) changes in the FSS-9 sum score at baseline and the predictors' influence on changes in the FSS-9 sum score (time trend) per year from baseline. The predictors' effect estimates and time trends estimate adjusted mean differences in the FSS-9 sum score

$A P R I$ aspartate transaminase to platelet ratio index, Cl confidence interval, CRP C-reactive protein, FSS-9 nine-item fatigue severity scale, eGFR estimated glomerular filtration rate, HIV human immunodeficiency virus, $k P a$ Kilopascal, OAT opioid agonist therapy

${ }^{1)}$ Age per 10 years was centred according to mean age (43 years) in the study sample at baseline. ${ }^{2)}$ Includes amphetamine or cocaine use. The educational level: highest level of education was coded 0-4 with 4 as the highest educational level. Unstable housing situation: living on the street, homeless shelter, or with family and friends at any time during the past 30 days prior to the health assessment. Debt difficulties: struggling with repaying current illegal and legal debt. Injecting substance use: Having injected substance during the past 12 months prior to the health assessment. Frequent use of substances: at least weekly during the past 12 months prior to the health assessment. Viral load of HCV: From -0.5 to 0.5 , where the range $\geq-0.5$ to $<0$ represents the low viral load (HCV PCR $<800,000$ IE/ $\mathrm{ml}$ ), and the range $\leq 0.5$ to $>0$ identifies the high viral load (HCV PCR $\geq 800,000 \mathrm{IE} / \mathrm{ml}$ ). Zero (0) defined patients without $\mathrm{HCV}$ infection

a) Statistically significant results when using Bonferroni corrected $p$-values ( $a_{\text {altered }}=0.05 / 41=0.0012$ )

use compared with those with less frequent or no use of these substances at baseline. For patients receiving methadone as an OAT opioid, the fatigue score was higher for females than males (seven points), for patients with frequent benzodiazepine use compared with those with less frequent or no use (six points), for patients with debt difficulties compared with those without difficulties with debt (five points), and for patients with a high versus a low viral load of HCV (32 points) at baseline. The latter finding suggesting an extreme difference between a high and a low viral load of HCV was surprising. Other studies assessing HCV viral load and correlation based on clinical and histological features have also not found HCV viral load to impact other related outcomes [26-28]. This finding is likely related to random variability within the data, and it should be interpreted with caution. Moreover, no sociodemographic and clinical factors were associated with clinically significant changes in the fatigue score from baseline to the following health assessments. 
In the present study, patients with SUD had a mean fatigue score (4.8) comparable to some of the most severe chronic diseases. In recent studies, patients infected with HIV or HCV, or those co-infected with both of them, have a mean FSS-9 score that ranged from 3.3 to $4.5[10,29,30]$. Patients with myasthenia gravis have reported a comparable fatigue score of 4.7 [31], and similarly, so have patients who have suffered from a stroke at least 6 months after the stroke onset (4.8) [32]. One can assume that a high prevalence of underlying mental disorders, extensive polysubstance use, and lower social status could have attributed to the high level of fatigue in the SUD population.

We found that females were weakly more fatigued than males. For both genders, patients with frequent use of benzodiazepines were weakly more fatigued than those with less frequent or no use. Recent studies evaluating fatigue in the general population and patients with chronic disorders have demonstrated similar gender differences in fatigue levels [15, 23, 31, 33]. Gender inequalities regarding household responsibilities and caring for the family have generally been highlighted as explanations for females' fatigue levels [34]. Additionally, females with SUD may be worse off than males in many domains. They may have less financial resources, experience more physical trauma caused by exchanging sex for drugs and money, and face more stigma related to family failures [34]. Moreover, in the general population and among patients with SUDs, females are more likely to use benzodiazepines than males, with a similar tendency found in different countries [2, 35-38]. Females' higher prevalence of anxiety disorders, sleeping disorders and the fact that they are more likely to seek medical care may contribute to more prescriptions of hypnotics and anxiolytics, such as benzodiazepines and $\mathrm{z}$ hypnotics [39-41]. One can believe that these medical, psychological, and social challenges may overall explain the gender gap concerning a higher fatigue level among females in the SUD population.

Our findings revealed that patients with frequent use of benzodiazepines were weakly more fatigued than those with less frequent or no use. Among patients with frequent use of stimulants, we found that they were less fatigued than those with less frequent or no use. These group differences were overall small. In the general population, previous studies assessing the effect of benzodiazepine use have suggested that patients using benzodiazepines have a lower quality of life, selfreported physical health, and more disability than those not using benzodiazepines [42, 43]. This is parallel with the higher fatigue levels shown in the present study. Furthermore, using stimulants, particularly illicit amphetamines, is generally associated with poor mental health and stimulant withdrawal symptoms in the SUD populations $[44,45]$. A temporary sense of better self-perceived mental health and fewer withdrawal symptoms may arise when consuming stimulant substances, which contributes to a temporary reduction of fatigue compared to the experience without stimulants. However, our trend analyses indicate that the fatigue levels remained stable over time when the frequent use of stimulants is persistent.

The present study showed no clear associations between fatigue and chronic infectious diseases or kidney disease. For patients with HBV, HIV or end-stage kidney disease, the low prevalence of HBV and HIV and a mean renal function within the normal range could explain why no associations with fatigue were detected. Furthermore, we are surprised that SUD patients with HCV infections did not demonstrate a higher fatigue level compared to patients with SUD without HCV infection, considering that the prevalence of fatigue is up to $70 \%$ in populations with $\mathrm{HCV}$ [9-11], which is considerably higher than in general population [23]. However, the large extent of polysubstance use in the present population $(75 \%)$ could have temporarily displaced the HCV infection's change on fatigue.

We found that $77 \%$ of patients using methadone as an OAT opioid and $67 \%$ of those using buprenorphine had severe fatigue symptoms. Four sociodemographic and clinical factors significantly changed fatigue levels among methadone users, while two factors influenced fatigue among those using buprenorphine. Methadone is a full opioid agonist associated with more euphoria and analgesia than the partial opioid agonist buprenorphine [46]. In a quantitative study evaluating patients' experience of using methadone and buprenorphine in OAT, unwanted physical effects, for example, over-sedation, were particularly pointed out in some methadone cases [47]. These effects might explain methadone users' high prevalence of severe fatigue symptoms and why more sociodemographic and clinical factors influenced methadone users' the fatigue levels than those using buprenorphine.

Overall, no single sociodemographic and clinical factor was associated with substantial changes in fatigue at baseline or over time in the SUD population. This signifies that fatigue was substantially constant between patients. However, the mean fatigue level significantly exceeded the threshold for severe fatigue in the SUD population, which underlies the importance of identifying patients who simultaneously have several sociodemographic and clinical risk factors for severe fatigue. Identifying these patients and treating the underlying causes of fatigue should be the way to reduce the fatigue in the population. 


\section{Strengths and limitations}

This study has several strengths. We have included 654 patients with SUD that usually are difficult to reach in health care. Of those, 225 patients were followed up by two or three annual health assessments, making longitudinal analyses possible. This study does, however, have some limitations. First, the patients were mainly recruited from outpatient clinics receiving OAT. The majority had opioid dependence, although this was often combined with other dependencies, which could affect the generalisability of our results to other SUD populations. Second, we had a prospective follow-up of only a third of those patients recruited at baseline. This also causes weakness in our results and makes it necessary to carefully interpret the longitudinal analyses. Third, due to clinical challenges, including systematic and patient delays, the annual health assessments were not precisely conducted one year after the previous health assessment. This may affect the interpretation of the predicted fatigue level changes from baseline. Fourth, due to data imputation and inclusion of up to 42 predictors in the linear mixed model analyses, there is a risk of Type I error. We dealt with this by presenting the Bonferroni corrections to all $p$-values in the analyses.

\section{Conclusion}

The present study shows a high symptom burden of fatigue among patients with SUDs, particularly among females, patients with debt difficulties, and those with extensive use of benzodiazepines. Identifying severe fatigue and considering fatigue in the follow-ups could help optimise SUD treatment for these patients. Policymakers could take this into consideration when planning health services.

\section{Supplementary Information}

The online version contains supplementary material available at https://doi. org/10.1186/s13011-020-00334-x

Additional file 1. The number of months from baseline to the second or third health assessment. No.: Number of patients; SD: Standard deviation; ref.: Reference. The table displays the number of patients with one, two, and three health assessments, including a nine-item Fatigue Severity Scale score. The table displays the time between baseline and the second and third health assessments.

Additional file 2. The US-English and the Norwegian versions of FSS-9. Description: Legends: FSS-9; Nine-item Fatique Severity Scale. All items in the FSS-9 are ranged as a Likert scale from 1 to 7 , where 1 indicates "strongly disagree" and 7 "strongly agree".

Additional file 3. Aspartate aminotransferase to platelet ratio index (APRI). Description: APRI: Aspartate aminotransferase to platelet ratio index. The figure displays the APRI score equation. The AST upper limit of normal range was defined as $45 \mathrm{IU} / \mathrm{L}$ (male) and $35 \mathrm{IU} / \mathrm{L}$ (female).

Additional file 4. Potential correlations between sociodemographic and clinical factors and fatigue. The figure shows that potential sociodemographic and clinical comorbidities may affect fatigue among patients with substance use disorders.

Additional file 5. Mean (SD) item scores for single items on the FSS-9 at baseline and follow-up. Legend: FSS-9: Nine-item Fatigue Severity Scale; I: Item; OAT: Opioid agonist therapy; SD: Standard Deviation. Follow-up: The FSS-9 score on the last health assessment during the study period among patients with two or more annual health assessments. Mean (SD) item scores for single items on the FSS-9 for patients using methadone or buprenorphine as OAT opioid.

Additional file 6. Linear mixed model of fatigue (FSS-9) adjusted for sociodemographic and clinical factors among patients receiving methadone as an OAT opioid at baseline $(N=209)$. Legends: APRI: Aspartate transaminase to platelet ratio index; $\mathrm{Cl}$ : Confidence interval; CRP: C-reactive protein; FSS-9: Nine-item Fatigue Severity Scale; eGFR: estimated glomerular filtration rate; HIV: Human Immunodeficiency virus; $\mathrm{kPa}$ Kilopascal; OAT: Opioid Agonist Therapy. ${ }^{1)}$ Age per 10 years was centred according to mean age (43 years) in the study sample at baseline. ${ }^{2)}$ Includes amphetamine or cocaine use. The OAT opioid ratio: a ratio between the received dose of OAT opioids per day divided by the expected mean dose of OAT opioids (buprenorphine $18 \mathrm{mg}$, buprenorphine-naloxone $18 / 4.5 \mathrm{mg}$ or methadone $90 \mathrm{mg}$ ). The educational level: highest level of education was coded $0-4$ with 4 as the highest educational level. Unstable housing situation: living on the street, homeless shelter, or with family and friends at any time in the past 30 days prior to the health assessment. Debt difficulties: struggling with repaying current illegal and legal debt. Injecting substance use: Having injected a substance in the past 12 months prior to the health assessment. Frequent use of substances: at least weekly during the past 12 months prior to the health assessment. Viral load of HCV: From -0.5 to 0.5 , where the range $\geq-0.5$ to $<0$ represents the low viral load (HCV $P C R<800,000 \mathrm{IE} / \mathrm{ml})$, and the range from $\leq 0.5$ to $>0$ identifies the high viral load (HCV PCR $\geq 800,000 \mathrm{IE} / \mathrm{ml}$ ). Zero (0) defined patients without $\mathrm{HCV}$ infection. When using the Bonferroni corrected $p$-values $\left(a_{\text {altered }}=\right.$ $0.05 / 43=0.0012$ ), the predictors did not affect the FSS-9 sum score significantly. The table displays a linear mixed model analysis (Restricted Maximum Likelihood regression) evaluating sociodemographic and clinical factors' (predictors) changes in the FSS-9 sum score at baseline and their influence on changes in the FSS-9 sum score (time trend) per year from baseline among patients receiving methadone as an OAT opioid. The predictors' effect estimates and time trends estimate adjusted mean differences in the FSS-9 sum score.

Additional file 7. Linear mixed model of fatigue (FSS-9) adjusted for sociodemographic and clinical factors among patients receiving buprenorphine as an OAT opioid at baseline $(N=321)$. Legends: APRI: Aspartate transaminase to platelet ratio index; $\mathrm{Cl}$ : Confidence interval; CRP: C-reactive protein; FSS-9: Nine-item Fatigue Severity Scale; eGFR: estimated glomerular filtration rate; HIV: Human Immunodeficiency virus; $\mathrm{kPa}$ : Kilopascal; OAT: Opioid Agonist Therapy. ${ }^{1)}$ Age per 10 years was centred according to mean age (43 years) in the study sample at baseline. ${ }^{2)}$ Includes amphetamine or cocaine use. The OAT opioid ratio: a ratio between the received dose of OAT opioids per day divided by the expected mean dose of OAT opioids (buprenorphine $18 \mathrm{mg}$, buprenorphine-naloxone $18 / 4.5 \mathrm{mg}$ or methadone $90 \mathrm{mg}$ ). The educational level: highest level of education was coded $0-4$ with 4 as the highest educational level. Unstable housing situation: living on the street, homeless shelter, or with family and friends at any time during the past 30 days prior to the health assessment. Debt difficulties: struggling with repaying current illegal and legal debt. Injecting substance use: Having injected a substance during the past 12 months prior to the health assessment. Frequent use of substances: at least weekly during the past 12 months prior to the health assessment. Viral load of HCV: From -0.5 to 0.5 , where the range $\geq-0.5$ to $<0$ represents the low viral load (HCV $P C R<800,000 \mathrm{IE} / \mathrm{ml})$, and the range $\leq 0.5$ to $>0$ identifies the high viral load (HCV PCR $\geq 800,000 \mathrm{IE} / \mathrm{ml}$ ). Zero (0) defined patients without HCV infection. When using the Bonferroni corrected $p$-values $\left(a_{\text {altered }}=0.05 /\right.$ $43=0.0012$ ), the predictors did not affect the FSS-9 sum score significantly. The table displays a linear mixed model analysis (Restricted Maximum Likelihood regression) evaluating sociodemographic and clinical factors' (predictors) changes in the FSS-9 sum score at baseline and their 
influence on changes in the FSS-9 sum score (time trend) per year from baseline among patients receiving buprenorphine as an OAT opioid. The predictors' effect estimates and time trends estimate adjusted mean differences in the FSS-9 sum score.

\section{Abbreviations}

APRI: Aspartate transaminase to platelet ratio index; $\mathrm{Cl}$ : Confidence interval; FSS-9: Nine-item Fatigue Severity Scale; HBV: Hepatitis B Virus; HCV: Hepatitis C Virus; HCV PCR: HCV Polymerase Chain Reaction; HIV: Human Immunodeficiency Virus; IQR: Interquartile Range; kPa: Kilopascal; OAT: Opioid Agonist Therapy; SUD: Substance Use Disorder; SD: Standard Deviation

\section{Acknowledgements}

We thank the devoted clinical staff for their enthusiasm during the planning of the project. We also thank Nina Elisabeth Eltvik and Christer Kleppe for their valuable help and input during the planning and preparation phases.

\section{Authors' contributions}

Jørn Henrik Vold and Rolf Gjestad have led the study design, analysis, and article preparation. Christer F. Aas, Fatemeh Chalabianloo, Svetlana Skurtveit, Else-Marie Løberg, Kjell Arne Johansson, and Lars Thore Fadnes have contributed by leading the study design, analysis, and article preparation. All authors have read and approved the final article.

\section{Funding}

This work was supported by The Norwegian Research Council (BEHANDLING, contract no 269855) and the Western Norway Regional Health Authority («Åpen prosjektstøtte») with the Department of Addiction Medicine, Haukeland University Hospital, Bergen, Norway as responsible institution. The funders had no role in the study design, data collection and analysis, decision to publish, or preparation of the manuscript. Two of the authors are funded from the research grant BEHANDLING related to the project INTROHCV from the Norwegian Research Council. The other authors are funded by their respective affiliations.

\section{Availability of data and materials}

No additional data are available due to data protection requirements.

\section{Ethics approval and consent to participate}

The study has been reviewed and approved by the Regional Ethical Committee for Health Research (REC) West, Norway (reference number: 2017/51/REK Vest, dated 29.03.2017/20.04.2017). Each patient provided written informed consent prior to enrolling in the study.

\section{Consent for publication}

Participants have consented for publication.

\section{Competing interests}

Not applicable.

\begin{abstract}
Author details
${ }^{1}$ Department of Addiction Medicine, Haukeland University Hospital, Jonas Lies vei 65, N-5021 Bergen, Norway. ${ }^{2}$ Department of Global Public Health and Primary Care, University of Bergen, Bergen, Norway. ${ }^{3}$ Department of Psychiatry, Haukeland University Hospital, Bergen, Norway. ${ }^{4}$ Department of Mental Disorders, Norwegian Institute of Public Health, Oslo, Norway. ${ }^{5}$ Norwegian Centre for Addiction Research, University of Oslo, Oslo, Norway. ${ }^{6}$ Department of Clinical Psychology, University of Bergen, Bergen, Norway.
\end{abstract}

\section{Accepted: 4 December 2020}

Published online: 14 December 2020

\section{References}

1. Lugoboni F, Mirijello A, Faccini M, Casari R, Cossari A, Musi G, Bissoli G, Quaglio G, Addolorato G. Quality of life in a cohort of high-dose benzodiazepine dependent patients. Drug Alcohol Depend. 2014;142:105-9.

2. Votaw VR, Geyer R, Rieselbach MM, McHugh RK. The epidemiology of benzodiazepine misuse: a systematic review. Drug Alcohol Depend. 2019; 200:95-114.
3. Morris L, Stander J, Ebrahim W, Eksteen S, Meaden OA, Ras A, Wessels A. Effect of exercise versus cognitive behavioural therapy or no intervention on anxiety, depression, fitness and quality of life in adults with previous methamphetamine dependency: a systematic review. Addict Sci Clin Pract. 2018;13(1):4.

4. Claborn KR, Meier E, Miller MB, Leffingwell TR. A systematic review of treatment fatigue among HIV-infected patients prescribed antiretroviral therapy. Psychol Health Med. 2015;20(3):255-65.

5. Ozyemisci-Taskiran O, Batur EB, Yuksel S, Cengiz M, Karatas GK. Validity and reliability of fatigue severity scale in stroke. Top. 2019;26(2):122-7.

6. Degenhardt L, Peacock A, Colledge S, Leung J, Grebely J, Vickerman P, Stone J, Cunningham EB, Trickey A, Dumchev K, et al. Global prevalence of injecting drug use and sociodemographic characteristics and prevalence of HIV, HBV, and HCV in people who inject drugs: a multistage systematic review. Lancet Glob Health. 2017;5(12):e1192-207.

7. Erman A, Sathya A, Nam A, Bielecki JM, Feld JJ, Thein HH, Wong WWL, Grootendorst $\mathrm{P}$, Krahn MD. Estimating chronic hepatitis $C$ prognosis using transient elastography-based liver stiffness: a systematic review and metaanalysis. J Viral Hepat. 2018;25(5):502-13.

8. Zacks SL, Fried MW. Hepatitis B and C and renal failure. Infect Dis Clin N Am. 2001;15(3):877-99.

9. Hassoun Z, Willems B, Deslauriers J, Nguyen BN, Huet PM. Assessment of fatigue in patients with chronic hepatitis $C$ using the fatigue impact scale. Dig Dis Sci. 2002;47(12):2674-81.

10. Scott J, Rosa K, Fu M, Cerri K, Peeters M, Beumont M, Zeuzem S, Evon DM, Gilles $L$. Fatigue during treatment for hepatitis $C$ virus: results of self-reported fatigue severity in two phase Ilb studies of simeprevir treatment in patients with hepatitis C virus genotype 1 infection. BMC Infect Dis. 2014;14:465.

11. Kallman J, O'Neil MM, Larive B, Boparai N, Calabrese L, Younossi ZM. Fatigue and health-related quality of life (HRQL) in chronic hepatitis $C$ virus infection. Dig Dis Sci. 2007;52(10):2531-9.

12. Jong E, Oudhoff LA, Epskamp C, Wagener MN, van Duijn M, Fischer S, van Gorp EC. Predictors and treatment strategies of HIV-related fatigue in the combined antiretroviral therapy era. Aids. 2010;24(10):1387-405.

13. Evon DM, Wahed AS, Johnson G, Khalili M, Lisker-Melman M, Fontana RJ, Sarkar S, Reeve BB, Hoofnagle JH. Fatique in patients with chronic hepatitis $B$ living in North America: results from the hepatitis B research network (HBRN). Dig Dis Sci. 2016;61(4):1186-96.

14. Saffari M, Pakpour AH, Al Zaben F, Koenig HG. Is there an association between health related quality of life, socio-demographic status and fatigue in patients with chronic hepatitis B? Acta Gastroenterol Belg. 2017;80(2):229-36.

15. Galland-Decker $C$, Marques-Vidal P, Vollenweider P. Prevalence and factors associated with fatigue in the Lausanne middle-aged population: a population-based, cross-sectional survey. BMJ Open. 2019;9(8):e027070.

16. Maglione MA, Raaen L, Chen C, Azhar G, Shahidinia N, Shen M, Maksabedian E, Shanman RM, Newberry S, Hempel S. Effects of medication assisted treatment (MAT) for opioid use disorder on functional outcomes: a systematic review. J Subst Abus Treat. 2018;89:28-51.

17. Krupp LB, LaRocca NG, Muir-Nash J, Steinberg AD. The fatigue severity scale. Application to patients with multiple sclerosis and systemic lupus erythematosus. Arch Neurol. 1989;46(10):1121-3.

18. Fukuda S, Takashima S, Iwase M, Yamaguti K, Kuratsune H, Watanabe Y. Development and validation of a new fatigue scale for fatigued subjects with and without chronic fatigue syndrome. In: Watanabe $Y$, Evengård $B$, Natelson BH, Jason LA, Kuratsune $\mathrm{H}$. (eds) Fatigue Science for Human Health. Tokyo: Springer; 2008.

19. Chalder T, Berelowitz G, Pawlikowska T, Watts L, Wessely S, Wright D, Wallace EP. Development of a fatigue scale. J Psychosom Res. 1993;37(2):147-53.

20. Rosa K, Fu M, Gilles L, Cerri K, Peeters M, Bubb J, Scott J. Validation of the fatigue severity scale in chronic hepatitis C. Health Qual Life Outcomes. 2014;12:90.

21. Siciliano M, Chiorri C, De Micco R, Russo A, Tedeschi G, Trojano L, Tessitore A. Fatigue in Parkinson's disease: Italian validation of the Parkinson fatigue scale and the fatigue severity scale using a Rasch analysis approach. Parkinsonism Relat Disord. 2019;65:105-10.

22. Fadnes LT, Aas CF, Vold JH, Ohldieck C, Leiva RA, Chalabianloo F, Skurtveit S, Lygren OJ, Dalgård O, Vickerman P, et al. Integrated treatment of hepatitis C virus infection among people who inject drugs: study protocol for a randomised controlled trial (INTRO-HCV). BMC Infect Dis. 2019;19(1):943.

23. Lerdal A, Wahl A, Rustøen T, Hanestad BR, Moum T. Fatigue in the general population: a translation and test of the psychometric properties of the 
Norwegian version of the fatigue severity scale. Scand J Public Health. 2005; 33(2):123-30.

24. Foucher J, Chanteloup E, Vergniol J, Castéra L, Le Bail B, Adhoute X, Bertet J, Couzigou $P$, de Lédinghen $V$. Diagnosis of cirrhosis by transient elastography (FibroScan): a prospective study. Gut. 2006;55(3):403-8.

25. Arpadi S, Fawzy A, Aldrovandi GM, Kankasa C, Sinkala M, Mwiya M, Thea DM, Kuhn L. Growth faltering due to breastfeeding cessation in uninfected children born to HIV-infected mothers in Zambia. Am J Clin Nutr. 2009;90(2): 344-53.

26. De Moliner L, Pontisso P, De Salvo GL, Cavalletto L, Chemello L, Alberti A. Serum and liver HCV RNA levels in patients with chronic hepatitis C: correlation with clinical and histological features. Gut. 1998;42(6):856-60.

27. Fanning L, Kenny E, Sheehan M, Cannon B, Whelton M, O'Connell J, Collins JK, Shanahan F. Viral load and clinicopathological features of chronic hepatitis C (1b) in a homogeneous patient population. Hepatology. 1999; 29(3):904-7.

28. Moatter T, Hussainy AS, Hamid S, Ahmad Z, Siddiqui S. Comparative analysis of viral titers and histologic features of Pakistani patients infected with hepatitis C virus type 3. Int J Infect Dis. 2002;6(4):272-6.

29. Kleefeld F, Heller S, Ingiliz P, Jessen H, Petersen A, Kopp U, Kraft A, Hahn K. Interferon-free therapy in hepatitis $\mathrm{C}$ virus (HCV) monoinfected and HCV/HIV coinfected patients: effect on cognitive function, fatigue, and mental health. J Neuro-Oncol. 2018;24(5):557-69.

30. Lee KA, Jong S, Gay CL. Fatigue management for adults living with HIV: a randomized controlled pilot study. Res Nurs Health. 2020;43(1):56-67.

31. Alekseeva TM, Gavrilov YV, Kreis OA, Valko PO, Weber KP, Valko Y. Fatigue in patients with myasthenia gravis. J Neurol. 2018;265(10):2312-21.

32. Naess H, Lunde L, Brogger J. The effects of fatigue, pain, and depression on quality of life in ischemic stroke patients: the Bergen stroke study. Vasc Health Risk Manag. 2012;8:407-13.

33. Sarkar S, Jiang Z, Evon DM, Wahed AS, Hoofnagle JH. Fatigue before, during and after antiviral therapy of chronic hepatitis C: results from the Virahep-C study. J Hepatol. 2012;57(5):946-52.

34. Arpa S. Women who use drugs: Issues, needs, responses, challenges and implications for policy and practice. European Monitoring Centre for Drugs and Drug Addiction (EMCDDA); 2017. https://www.emcdda.europa.eu/ system/files/attachments/6235/EuropeanResponsesGuide2017_ BackgroundPaper-Women-who-use-drugs.pdf. Accessed 27 Nov 2020.

35. Skurtveit S, Sakshaug S, Hjellvik V, Berg C, Handal M. Use of addictive drugs in Norway 2005-2013 (Norsk: Bruk av vanedannende legemidler i Norge 2005-2013). Norwegian Institute of Public Health; 2014. https://www.fhi.no/ globalassets/dokumenterfiler/rapporter/2014/bruk-av-vanedannendelegemidler-pdf.pdf. Accessed 27 Nov 2020.

36. Airagnes $\mathrm{G}$, Lemogne $\mathrm{C}$, Renuy $\mathrm{A}$, Goldberg $\mathrm{M}$, Hoertel $\mathrm{N}$, Roquelaure $\mathrm{Y}$, Limosin F, Zins M. Prevalence of prescribed benzodiazepine long-term use in the French general population according to sociodemographic and clinical factors: findings from the CONSTANCES cohort. BMC Public Health. 2019;19(1):566.

37. Petitjean $S$, Ladewig D, Meier CR, Amrein R, Wiesbeck GA. Benzodiazepine prescribing to the Swiss adult population: results from a national survey of community pharmacies. Int Clin Psychopharmacol. 2007;22(5):292-8.

38. Bachhuber MA, Hennessy S, Cunningham CO, Starrels JL. Increasing benzodiazepine prescriptions and overdose mortality in the United States, 1996-2013. Am J Public Health. 2016;106(4):686-8.

39. McLean CP, Asnaani A, Litz BT, Hofmann SG. Gender differences in anxiety disorders: prevalence, course of illness, comorbidity and burden of illness. J Psychiatr Res. 2011;45(8):1027-35.

40. Krishnan V, Collop NA. Gender differences in sleep disorders. Curr Opin Pulm Med. 2006;12(6):383-9.

41. Wang Y, Hunt K, Nazareth I, Freemantle N, Petersen I. Do men consult less than women? An analysis of routinely collected UK general practice data. BMJ Open. 2013;3(8):e003320.

42. Abrahamsson T, Hakansson A. Nonmedical prescription drug use (NMPDU) in the Swedish general population--correlates of analgesic and sedative use. Subst Use Misuse. 2015;50(2):148-55.

43. Ford JA, Hinojosa MS, Nicholson HL. Disability status and prescription drug misuse among U.S. adults. Addict Behav. 2018;85:64-9.

44. McKetin R, Leung J, Stockings E, Huo Y, Foulds J, Lappin JM, Cumming C, Arunogiri S, Young JT, Sara G, et al. Mental health outcomes associated with of the use of amphetamines: a systematic review and meta-analysis. EClinicalMedicine. 2019;16:81-97.
45. Pennay AE, Lee NK. Putting the call out for more research: the poor evidence base for treating methamphetamine withdrawal. Drug Alcohol Rev. 2011;30(2):216-22.

46. Whelan PJ, Remski K. Buprenorphine vs methadone treatment: a review of evidence in both developed and developing worlds. J Neurosci Rural Pract. 2012;3(1):45-50.

47. Gryczynski J, Jaffe JH, Schwartz RP, Dušek KA, Gugsa N, Monroe CL, O'Grady KE, Olsen YK, Mitchell SG. Patient perspectives on choosing buprenorphine over methadone in an urban, equal-access system. Am J Addict. 2013;22(3): 285-91.

\section{Publisher's Note}

Springer Nature remains neutral with regard to jurisdictional claims in published maps and institutional affiliations.
Ready to submit your research? Choose BMC and benefit from:

- fast, convenient online submission

- thorough peer review by experienced researchers in your field

- rapid publication on acceptance

- support for research data, including large and complex data types

- gold Open Access which fosters wider collaboration and increased citations

- maximum visibility for your research: over $100 \mathrm{M}$ website views per year

At BMC, research is always in progress.

Learn more biomedcentral.com/submissions 